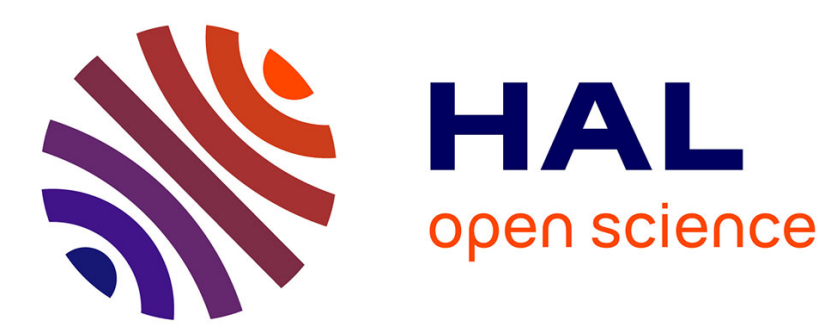

\title{
The hyperfine structure in the rotational spectra of bromofluoromethane: Lamb-dip technique and quantum-chemical calculations
}

Gabriele Cazzoli, Cristina Puzzarini, Stella Stopkowicz, Jürgen Gauss

\section{To cite this version:}

Gabriele Cazzoli, Cristina Puzzarini, Stella Stopkowicz, Jürgen Gauss. The hyperfine structure in the rotational spectra of bromofluoromethane: Lamb-dip technique and quantum-chemical calculations. Molecular Physics, 2008, 106 (09-10), pp.1181-1192. 10.1080/00268970802056052 . hal-00513193

\author{
HAL Id: hal-00513193 \\ https://hal.science/hal-00513193
}

Submitted on 1 Sep 2010

HAL is a multi-disciplinary open access archive for the deposit and dissemination of scientific research documents, whether they are published or not. The documents may come from teaching and research institutions in France or abroad, or from public or private research centers.
L'archive ouverte pluridisciplinaire HAL, est destinée au dépôt et à la diffusion de documents scientifiques de niveau recherche, publiés ou non, émanant des établissements d'enseignement et de recherche français ou étrangers, des laboratoires publics ou privés. 


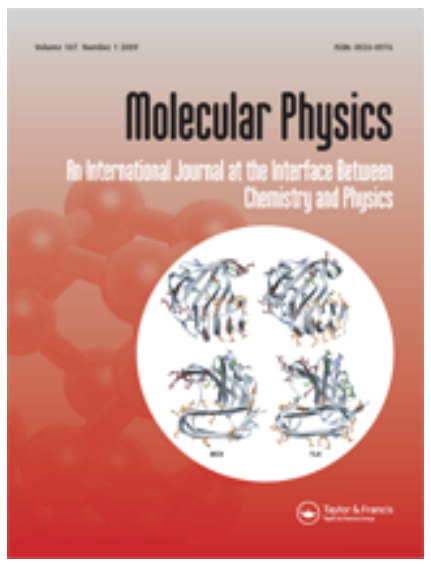

The hyperfine structure in the rotational spectra of bromofluoromethane: Lamb-dip technique and quantumchemical calculations

\begin{tabular}{|c|c|}
\hline Journal: & Molecular Physics \\
\hline Manuscript ID: & TMPH-2008-0020 \\
\hline Manuscript Type: & Full Paper \\
\hline $\begin{array}{r}\text { Date Submitted by the } \\
\text { Author: }\end{array}$ & 27-Jan-2008 \\
\hline Complete List of Authors: & $\begin{array}{l}\text { Cazzoli, Gabriele; Università di Bologna, Dip. Chimica "G. } \\
\text { Ciamician" } \\
\text { Puzzarini, Cristina; Università di Bologna } \\
\text { Stopkowicz, Stella; Universität Mainz } \\
\text { Gauss, Jürgen; Universität Mainz }\end{array}$ \\
\hline Keywords: & $\begin{array}{l}\text { bromofluoromethane and deuterated species, rotational } \\
\text { spectroscopy and Lamb-dip technique, nuclear quadrupole coupling } \\
\text { and spin-rotation, quantum-chemical calculations, relativistic effects }\end{array}$ \\
\hline \multicolumn{2}{|c|}{$\begin{array}{l}\text { Note: The following files were submitted by the author for peer review, but cannot be converted } \\
\text { to PDF. You must view these files (e.g. movies) online. }\end{array}$} \\
\hline hfs-ch2fbr.tex & \\
\hline
\end{tabular}

\section{S ScholarONE Manuscript Central}




\author{
Gabriele Cazzoli, * Cristina Puzzarini, \\ Dipartimento di Chimica "G. Ciamician", Università di Bologna, Via Selmi 2, \\ I-40126 Bologna, Italy \\ Stella Stopkowicz, Jürgen Gauss* \\ Institut für Physikalische Chemie, Universität Mainz, D-55099 Mainz, Germany
}

\begin{abstract}
The hyperfine structure in the rotational spectra of six isotopic species of bromofluoromethane, namely $\mathrm{CH}_{2}{ }^{79} \mathrm{BrF}, \mathrm{CH}_{2}{ }^{81} \mathrm{BrF}, \mathrm{CDH}^{79} \mathrm{BrF}, \mathrm{CDH}^{81} \mathrm{BrF}, \mathrm{CD}_{2}{ }^{79} \mathrm{BrF}$, and $\mathrm{CD}_{2}{ }^{81} \mathrm{BrF}$, has been investigated using the Lamb-dip technique in the submillimeterwave frequency range. Measurements as well as assignment procedures have been supported by high-level quantum-chemical calculations of the hyperfine parameters at the coupled-cluster level. For all species, the accuracy of the determined rotational and centrifugal distortion constants as well as the bromine quadrupole-coupling constants have been improved with respect to available literature data, whereas the full bromine quadrupole-coupling tensor for the monodeuterated species and the bromine spin-rotation constants for all isotopologues have been determined for the first time.
\end{abstract}

Key words: bromofluoromethane, deuterated species, rotational spectroscopy, Lamb-dip technique, nuclear quadrupole coupling, spin-rotation, quantum-chemical calculations, relativistic effects

\footnotetext{
* corresponding authors

Email addresses: gabriele.cazzoli@unibo.it (Gabriele Cazzoli,), gauss@uni-mainz.de (Jürgen Gauss).

1 Special Issue: Twentieth Colloquium on High Resolution Molecular Spectroscopy, Dijon 2-7 September 2007
} 


\section{Introduction}

The determination of (hyper)fine parameters, such as quadrupole-coupling, spin-spin coupling, and spin-rotation constants, is one of the aims of highresolution rotational spectroscopy. These parameters are relevant not only from a spectroscopic point of view, but also from a physical and/or chemical viewpoint, as they might provide detailed information on the chemical bond, structure, ... [1]. In addition, hyperfine structures are so characteristic that their analysis may help in assigning rotational spectra of unknown species (see for instance [2]). Nevertheless, the experimental determination of hyperfine constants can be a challenge because of either the lack of reliable estimates or the complexity of the hyperfine structure itself. Therefore, it is important to have the opportunity to rely on good predictions for such parameters. These can be provided by quantum chemistry. In fact, it is well known that nowadays spectroscopic parameters, and among those also the hyperfine constants, can be accurately calculated by means of highly correlated methods in conjunction with large basis sets (see for example [2-6]). One important issue to be taken into consideration is whether the same good accuracy can be reached when atoms as large as bromine are involved. In fact, in these cases on one hand relativistic effects may become relevant (see, for example, Refs. [7-10]), on the other hand computations are more expensive and this may preclude the use of either highly accurate methods or large basis sets.

Bromofluoromethane provides a good test case for checking the accuracy of experimental as well as theoretical investigations. To this purpose, in the present work the hyperfine structures of the rotational spectra of six isotopic species of bromofluoromethane, namely $\mathrm{CH}_{2}{ }^{79} \mathrm{BrF}, \mathrm{CH}_{2}{ }^{81} \mathrm{BrF}, \mathrm{CDH}^{79} \mathrm{BrF}, \mathrm{CDH}^{81} \mathrm{BrF}$, $\mathrm{CD}_{2}{ }^{79} \mathrm{BrF}$, and $\mathrm{CD}_{2}{ }^{81} \mathrm{BrF}$, have been investigated by means of the Lambdip technique. The experimental investigation has been supported by highly accurate calculations of hyperfine parameters, i.e., spin-rotation and nuclear quadrupole-coupling constants. It will be shown that even for a pentatomic molecule containing a third-row element reliable estimates can be obtained in quantum-chemical investigations, though relativistic effects are found to be quite substantial in the case of the bromine electric-field gradient and quadrupole-coupling tensor.

Bromofluoromethane has already been studied in our laboratory as the subject of a project aiming at a full characterization of its various isotopologues in order to determine its structural and spectroscopic properties [11-14]. $\mathrm{CH}_{2} \mathrm{BrF}$ has attracted our attention because halogen-containing species have some connection with stratospheric ozone depletion [15-17], but reliable experimental data for many halocarbons are still unavailable, especially for those containing bromine. Although no information on the environmental relevance is available for this molecule, it could affect the composition of the atmosphere since 
bromofluoromethane can potentially contribute to the destruction of stratospheric ozone. In fact, its ozone depletion potential (ODP) is 0.73 with respect to CFC-116 [18].

\section{Experimental details}

The rotational spectra of six isotopic species of $\mathrm{CH}_{2} \mathrm{BrF}$ have been investigated at sub-Doppler resolution. More precisely, the mono- and bideuterated species of bromofluoromethane, $\mathrm{CDH}^{79} \mathrm{BrF}, \mathrm{CDH}^{81} \mathrm{BrF}, \mathrm{CD}_{2}{ }^{79} \mathrm{BrF}$, and $\mathrm{CD}_{2}{ }^{81} \mathrm{BrF}$, as well as the two main isotopologues, $\mathrm{CH}_{2}{ }^{79} \mathrm{BrF}$ and $\mathrm{CH}_{2}{ }^{81} \mathrm{BrF}$, have been considered. Enriched samples have been employed (see Refs. [12-14] for details).

The Lamb-dip technique has been exploited throughout in order to better resolve the fine structure due to the bromine nucleus in the rotational transitions and, for deuterated species, to try to resolve that due to deuterium. To this purpose, as shown in Ref. [19], a frequency modulated computer-controlled spectrometer has been employed using a conventional free space cell. To increase the sensitivity of the spectrometer as well as of the Lamb-dip effect the radiation path has been doubled (see Ref. [20]). A detailed description of the spectrometer is given in Ref. [21], whereas our experimental set up for performing sub-Doppler resolution spectroscopy has been described in some previous papers [22-25]. Therefore, here, we only report the main details related to the present investigation. The submillimeter-wave sources employed are frequency multipliers driven by gunn diode oscillators, and the frequency range considered is mainly $350-550 \mathrm{GHz}$. The source is phase-locked to a Rubidium frequency standard, and the frequency modulation is obtained by sine-wave modulating the $73 \mathrm{MHz}$ local oscillator of the synchronization loop. A liquid He cooled InSb detector has been used and its output is processed by means of a Lock-in amplifier tuned at twice the modulation frequency, so that the second derivative of the natural line profile has been recorded.

The Lamb-dip measurements have been carried out at pressures of $0.3-1$ mTorr. The modulation depth used has been adjusted in the ranges of 8 to $50 \mathrm{kHz}$ according to the experimental conditions and the transitions under consideration. The low values of working pressure and of modulation depth have been chosen in order to minimize as much as possible the dip widths, and thus to improve the resolution. The modulation frequency has been kept fixed at $1.666 \mathrm{kHz}$.

Figure 1a provides as an example a portion of the spectrum of the sample containing mono- and bideuterated bromofluoromethane (roughly $30 \%$ of both, for details see Ref. [14]) recorded at Doppler (black) and sub-Doppler (red) 
resolution. This figure allows us to emphasize how the fine structure is better resolved by employing the Lamb-dip technique and how many hyperfine components are actually present. Furthermore, Figure $1 \mathrm{~b}$ shows that it is possible to even observe the Lamb-dip components of a rotational transition with $J$ equal to 64 of $\mathrm{CH}_{2}{ }^{79} \mathrm{BrF}$ in such crowded spectra and for samples containing only $\sim 30 \%$ of the main isotopic species (i.e., $\mathrm{CH}_{2}{ }^{79} \mathrm{BrF}$ and $\mathrm{CH}_{2}{ }^{81} \mathrm{BrF}$ ).

\section{Computational details}

The experimental determination has been supported by quantum-chemical calculations of the nuclear quadrupole-coupling and spin-rotation tensors of ${ }^{79} \mathrm{Br}$ and ${ }^{81} \mathrm{Br}$. In addition, computations have also been performed, for predictive purposes, for the nuclear quadrupole-coupling constants of deuterium and the spin-rotation constants of fluorine and hydrogen.

For nuclei with a quadrupole moment, i.e., those with nuclear spin quantum number $I_{K} \geq 1$, the main contribution to the (hyper)fine structure of rotational spectra arises from the interaction of the nuclear quadrupole moment (defined for nucleus $K$ as $-e Q_{K}$ ) and the electric-field gradient at that nucleus $\boldsymbol{V}^{K}[26]$ :

$$
\mathbf{H}_{Q}=\frac{1}{2} \sum_{K} \frac{-e Q_{K}}{I_{K}\left(2 I_{K}-1\right)} \boldsymbol{I}_{K} \cdot \boldsymbol{V}^{K} \cdot \boldsymbol{I}_{K}
$$

The elements of the nuclear quadrupole-coupling tensor for the nucleus $K$ are then defined as $\chi_{i j}=-e Q_{K} V_{i j}^{K} / \hbar$, where $i, j$ refer to the inertial axes $a, b$, and/or $c$.

Since bromofluoromethane has a symmetry plane $\left(C_{s}\right.$ symmetry) and Br lies on this plane, its inertial nuclear quadrupole-coupling tensor has only one off-diagonal nonzero element, namely $\chi_{a b}$ :

$$
\chi^{I}=\left(\begin{array}{ccc}
\chi_{a a} & \chi_{a b} & 0 \\
\chi_{a b} & \chi_{b b} & 0 \\
0 & 0 & \chi_{c c}
\end{array}\right) .
$$

However, in the case of the monodeuterated species, the $C_{s}$ symmetry is lost and all off-diagonal elements are nonzero. For the deuterium quadrupolecoupling tensor all off-diagonal terms are nonzero, as the site symmetry in this case is always $C_{1}$.

As far as the spin-rotation interactions are concerned, Flygare derived a formulation in terms of a second-rank tensor $\boldsymbol{C}$ coupled with the rotational $\boldsymbol{J}$ 
and nuclear spin $\boldsymbol{I}$ momenta [27]:

$$
\mathbf{H}_{S R}=\sum_{K} \boldsymbol{I}_{K} \cdot \boldsymbol{C}_{K} \cdot \boldsymbol{J},
$$

where the sum over $K$ runs over all nuclei of the molecule. As well-known, only the nuclei with $I \geq 1 / 2$ have nonzero spin-rotation tensors, that means in our case Br, F, H and/or D. Note that we adopt in Eq. (3) a sign convention for the spin-rotation constants opposite to that used by Flygare [27]. Each element of the spin-rotation tensor has an electronic and a nuclear contribution. The electronic contribution can be evaluated as second derivative of the electronic energy with respect to the rotational angular momentum $\boldsymbol{J}$ and the nuclear spin $\boldsymbol{I}_{K}$ [28]. This is efficiently done using analytic secondderivative techniques [29-32]. Perturbation-dependent basis functions (often referred to as rotational London Atomic Orbitals [33]) have been employed to improve basis-set convergence and to avoid an unphysical origin dependence. The nuclear contribution depends only on the molecular geometry.

The electric-field gradient as well as spin-rotation tensor calculations have been performed at the coupled cluster (CC) level of theory [34]. More precisely, the CC singles and doubles (CCSD) approximation augmented by a perturbative treatment of triple excitations $(\operatorname{CCSD}(\mathrm{T}))[35]$ has been used. The core-valence correlation consistent cc-pCVnZ bases $[36,37]$, with $n=\mathrm{T}$, Q, have been employed, and all electrons have been considered in the electron-correlation treatment. To be more specific, the CCSD $(\mathrm{T}) / \mathrm{cc}$-pCVQZ level of theory has been employed in all electric-field gradient computations, whereas the CCSD(T)/ccpCVTZ level has been adopted in the calculations of the spin-rotation interaction constants. In regard to the latter, only for the $\mathrm{CH}_{2}{ }^{79} \mathrm{BrF}$ isotopologue CCSD(T)/cc-pCVQZ calculations have been performed.

Relativistic effects on the electric-field gradient at the bromine nucleus and thus on the bromine quadrupole-coupling tensor have been taken into account using a perturbative scheme. The corresponding corrections have been obtained at the CC level using second-order direct perturbation theory (DPT2) [38]. With the relativistic energy correction in DPT2 given as a first derivative of the energy [39], the DPT2 corrections to first-order properties are obtained as second derivatives of the energy with respect to the corresponding perturbation parameters $[40,41]$ and are best computed using analytic second-derivative techniques [41]. The calculations have been carried out at the $\operatorname{CCSD}(\mathrm{T})$ level using large uncontracted basis sets (cc-pVQZ-unc) obtained by uncontracting the corresponding cc-pVQZ sets. The accuracy of the DPT2 treatment of relativistic effects on the electric-field gradient at the bromine nucleus has been checked at the Hartree-Fock (HF) level in the case of $\mathrm{HBr}$ by comparison with available four-component Dirac HF results from the literature [10] and the agreement is found (with a deviation of less than 1\%) more than satisfactory [41]. 
The nuclear qudrupole-moment values have been taken in the case of deuterium from Ref. [50] and in the case of the two bromine isotopes from Ref. [43].

Zero-point vibrational (ZPV) corrections to electric-field gradient and spinrotation constants have been obtained using the perturbational approach described in Ref. [44] for NMR shielding tensors and which has already been applied in Refs. [5,6] for spin-rotation constants. The vibrational corrections have been computed at the second-order Møller-Plesset perturbation theory (MP2) [45] level in conjunction with the cc-pCVTZ basis set, correlating again all electrons. The vibrational corrections, defined as the difference between the vibrationally averaged and the equilibrium values, have then added to the $\operatorname{CCSD}(\mathrm{T})$ results to derive our best theoretical estimates of the ground state data, values that can be directly compared to experiment.

All calculations (except those for the zero-point corrections which have been performed at the corresponding equilibrium geometries) have been carried out at the best estimated equilibrium structure [46] given in Ref. [14].

The Mainz-Austin-Budapest version of the ACESII program package [47] has been used for all computations reported in the present paper. Some of the calculations have been carried out using the recently developed parallel version [48] of ACESII.

\section{Analysis of the Spectra}

If only the bromine nucleus is considered, the various hyperfine components result from the $\Delta F=+1,0,-1$ transitions, where $F$ is the quantum number originating from the coupling scheme $F=J+I_{B r}$.

The corresponding Hamiltonian can be written as:

$$
\mathbf{H}=\mathbf{H}_{R O T}+\mathbf{H}_{Q}+\mathbf{H}_{S R},
$$

where $\mathbf{H}_{R O T}$ accounts for the pure rotational part [1], and $\mathbf{H}_{Q}$ as well as $\mathbf{H}_{S R}$ have been already defined in the previous section (Eqs. (1) and (3), respectively), with the sum restricted to the bromine nucleus.

Concerning the analysis of the spectra, we note that the hyperfine component frequencies have been determined either by fitting the experimental data points to a parabolic function or by a line profile analysis (see Ref. [21]), with the latter used for distorted dips. For all isotopologues considered the determined hyperfine components of the rotational transitions have an estimated accuracy of $1.5 \mathrm{kHz}$. On the whole, a total of 192, 266, 140, 182, 204, 224 hyperfine components have been assigned for $\mathrm{CH}_{2}{ }^{79} \mathrm{BrF}, \mathrm{CH}_{2}{ }^{81} \mathrm{BrF}, \mathrm{CDH}^{79} \mathrm{BrF}$, 
$\mathrm{CDH}^{81} \mathrm{BrF}, \mathrm{CD}_{2}{ }^{79} \mathrm{BrF}$, and $\mathrm{CD}_{2}{ }^{81} \mathrm{BrF}$, respectively. The transition frequencies are not reported here but are available from the authors upon request.

For all the isotopic species considered in the present study, the analysis has been carried out by comparing graphical representations of the calculated spectra to the observed ones [49]. For predicting the hyperfine pattern of the rotational spectra due to the bromine nucleus, we generally used the experimental rotational constants (from Refs. [12,13]) together with the computed hyperfine (quadrupole-coupling and spin-rotation, from this work) parameters. Going into details, it has to be noted that the experimental investigation reported in Ref. [14] and this study were started at the same time; thus, prior to them only the full bromine quadrupole-coupling tensors of $\mathrm{CH}_{2}{ }^{79} \mathrm{BrF}$ and $\mathrm{CH}_{2}{ }^{81} \mathrm{BrF}$ were known. Therefore, while for the main isotopologues it was only necessary to use computed values for the spin-rotation tensor, for the monoand bideuterated species it was essential to use also the computed data for the quadrupole-coupling tensor. However, this data could be improved by using the following empirical scaling procedure:

$$
\chi_{\text {scal }}^{i}=\chi_{\text {calc }}^{i} \times\left(\chi_{\text {exp }}^{p} / \chi_{\text {calc }}^{p}\right),
$$

where $i$ and $p$ refer to the isotopic substituted and the parent molecule, respectively, and scal, exp, and calc denote the scaled, experimental and calculated values, respectively. $\mathrm{CH}_{2}{ }^{79} \mathrm{BrF}$ and $\mathrm{CH}_{2}{ }^{81} \mathrm{BrF}$ have been used as parent molecule for isotopologues containing ${ }^{79} \mathrm{Br}$ and ${ }^{81} \mathrm{Br}$, respectively. Furthermore, in the case of the monodeuterated species, the $\chi_{a c}$ and $\chi_{b c}$ off-diagonal elements are nonzero and could not be scaled.

Figure 2 provides an example that deserves a few comments. First of all, it has to be noted that the sub-Doppler (red) and Doppler (black) $\Delta F=$ $0(F=49 / 2,51 / 2,53 / 2,55 / 2)$ resolved components of the $26_{6,20} \leftarrow 26_{5,21}$ and $26_{6,21} \leftarrow 26_{5,22}$ rotational transitions of $\mathrm{CH}_{2}{ }^{79} \mathrm{BrF}$ are depicted. The figure thus shows that the central frequency can appear different when changing the spectra resolution: in this case, the apparent difference is about $40-50 \mathrm{kHz}$. Such a difference can be only recovered by performing a line profile analysis of the Doppler limited spectrum. However, it is clear that the Lamb-dip technique is needed when highly accurate transition frequencies are required.

Concerning the deuterated species, it should be mentioned that, by making use of our theoretical predictions, we tried to resolve the hyperfine structure due to deuterium. Unfortunately, the splitting due to the deuterium quadrupole coupling is almost negligible, i.e., of the order of a few $\mathrm{kHz}$. In the frequency range covered by our spectrometer $(80-800 \mathrm{GHz})$, there are only a few transitions for which a larger splitting (i.e., $15-20 \mathrm{kHz}$ ) has been predicted. We tried to resolve them, but, as shown in figure 3 , we did not succeeded. To be more specific, in figure 3 a portion of the Lamb-dip spectrum of the $J=2_{2,0} \leftarrow 1_{1,1}$ rotational transition of $\mathrm{CHD}^{79} \mathrm{BrF}$ is shown together with two calculated spec- 
tra which have been obtained by using different values of modulation depth, resulting in different resolutions. In case (a) a modulation depth of $20 \mathrm{kHz}$ has been employed for the simulation, whereas in (b) a modulation depth of $25 \mathrm{kHz}$ has been used. From the comparison it is clear that the two hyperfine components are hardly resolvable in the experimental spectrum due to a poor signal to noise ratio.

\section{Results and Discussion}

The determined frequencies have been included in least-squares fits in which each line frequency has been weighted proportional to the inverse square of its experimental uncertainty. For unresolved hyperfine components, the calculated frequencies have been determined using intensity-weighted averages. In addition to the hyperfine components measured in the present work, the rotational transitions from Refs. [12-14] have also been included in the analysis. The fits have been carried out with Pickett's SPCAT/SPFIT suite of programs [50], employing Watson's A-reduced Hamiltonian in the $I^{r}$ representation [51]. The results are reported in Tables 1 to 3, where the spectroscopic parameters are compared with the corresponding theoretical values.

From Tables 1 to 3, it is first of all evident that we were able to improve the accuracy of all the spectroscopic parameters with respect to those given in Refs. [12-14]. There is a slight improvement (error reduced by roughly one third) in the rotational constants for all the species except the monodeuterated ones for which even a significantly larger improvement is noted (i.e., by approximately one order of magnitude). Concerning the centrifugal distortion constants, most of them are improved by about one order of magnitude and in some cases it was even possible to determine new, so far unavailable constants. Concerning the hyperfine parameters, let us first consider the bromine quadrupole-coupling tensor. While generally only minor improvements are observed here, it should be noted that the off-diagonal terms $\chi_{a c}$ and $\chi_{b c}$ (in addition to $\chi_{a b}$ ) are determined for the first time for the monodeuterated isotopologues. The same is also the case for some of the $\chi_{a a}^{J}$ and $\chi_{a a}^{K}$ constants which account for the $J$-dependence and $K$-dependence of $\chi_{a a}$. With respect to spin-rotation interactions, the diagonal elements of the bromine tensor have been determined for the first time.

Since theoretical predictions of the hyperfine parameters have been used to guide the recording as well as the assignment of the Lamb-dip spectra, the comparison between experimental and theoretical values deserves a brief discussion. At a first sight, one may note an overall good agreement between experiment and theory (see Tables 1 to 3 ). 
In the case of the bromine quadrupole-coupling tensor, the remaining discrepancy is about 1 to $2 \%$. Only for the off-diagonal elements $\chi_{a c}$ and $\chi_{b c}$, which are non-zero only for the monodeuterated isotopic species, larger relative errors are observed, but these off-diagonal elements are (unlike the diagonal elements and $\chi_{a b}$ ) rather small and affected by large experimental uncertaintities. Table 4 gathers the various contribution to our final best theoretical values for the bromine quadrupole-coupling tensors. We note that relativistic effects are rather large (i.e. of the order of $6 \%$ ) and thus essential to obtain a satisfactory agreement between theory and experiment. It should be also noted that in comparison to experiment the computed values are in all cases too large in absolute terms, thus indicating to a systematic error as source for the remaining discrepancies. The apparent good agreement between theory and experiment found in Ref. [14] based on non-relativistic quantum-chemical calculations only turned out to be due to the use of old values for the nuclear quadrupole moment. These values have been in the mean time revised [43], and the use of the current values clearly reveals the need for inclusion of relativistic effects. Zero-point vibrational corrections to the bromine quadrupole-coupling tensor on the other hand are rather small and to amount to less than $1 \%$. The overall reached agreement between theory and experiment can be considered satisfactory. The remaining deviations can be probably attributed to basis-set truncation errors in the non-relativistic calculations as most likely source and also to the uncertainty (about 1\%) in the experimental values for the nuclear quadrupole moments.

The determination of the full bromine nuclear quadrupole coupling tensors in the inertial axes system (see Tables 1 to 3) allows a discussion of how these tensor differ for the different isotopologues considered in the present study. First of all, we note that it is only meaningful to directly compare those containing the same Br isotope. Furthermore, a comparison in the inertial axes system is not meaningful and it is essential to compare these tensors in their own principal axes system. In this way, we are able to distinguish between the effect which result from the different vibrational contributions to $\chi$ and those arising from the different orientation of the principal axes system. In Table 5, we thus report for all investigated species the $\chi_{x x}, \chi_{y y}$, and $\chi_{z z}$ values obtained by diagonalizing the nuclear quadrupole-coupling tensor. We note that the comparison of theory and experiment is again very satisfactory and that the same trends are observed in the calculations as in the experimental investigation.

In Table 6 the computed values of the deuterium quadrupole-coupling constants are summarized for both mono- and bideuterated species $\left(\mathrm{CDH}^{79} \mathrm{BrF}\right.$, $\mathrm{CDH}^{81} \mathrm{BrF}, \mathrm{CD}_{2}{ }^{79} \mathrm{BrF}$, and $\left.\mathrm{CD}_{2}{ }^{81} \mathrm{BrF}\right)$. As seen from Table 6, these coupling constants are small (being almost all smaller than $100 \mathrm{kHz}$ ). On the basis of the results reported in Tables $1-3$ and the discussion above, the relative accuracy of these constants is expected to be of the order of $1 \%$. Unlike 
for bromine, relativistic effects play no significant role for the corresponding deuterium quadrupole couplings.

With respect to the spin-rotation constants, it has to be noted that their magnitude is rather small, i.e., they amount to about 5 to $15 \mathrm{kHz}$. A discussion of relative errors thus is more or less meaningless, and it s more interesting to focus here on the absolute accuracy. From Tables 1 to 3 it is evident that the deviations between experiment and theory vary from $0.1 \mathrm{kHz}$ up to, in worst cases, $\sim 1 \mathrm{kHz}$. In most cases these discrepancies are smaller than the experimental uncertainties, and in all cases they are smaller than three times the standard deviation. The computational accuracy can be inferred from comparisons between CCSD(T)/cc-pCVTZ and CCSD(T)/cc-pCVQZ results. The differences are in the range of 0.1 to $0.3 \mathrm{kHz}$, thus indicating that the use of a core-polarized triple-zeta basis can be considered sufficient. Unlike for the quadrupole-coupling constants, zero-point vibrational corrections are with 0.1 to $2 \mathrm{kHz}$ of some significance, i.e., in the range of 4 to $10 \%$.

Finally, for the sake of completeness, we also report in Table 7 the spinrotation constants of the fluorine and hydrogen nuclei. One may note that these parameters are very small and experimentally not determinable by the available instrumentation. Concerning the deuterium spin-rotation constants, we do not report the corresponding values (all are smaller than $0.5 \mathrm{kHz}$ ) even though they are available from the calculations.

\section{Conclusions}

The present study is an example for the successful interplay of theory and experiment and demonstrates the importance of accurate theoretical predictions in particular in the case of crowded or complicated spectra. In the present investigation the Lamb-dip technique has been used to resolve the hyperfine structure due to the bromine nucleus in the rotational spectra of six isotopic species of bromofluoromethane, namely $\mathrm{CH}_{2}{ }^{79} \mathrm{BrF}, \mathrm{CH}_{2}{ }^{81} \mathrm{BrF}, \mathrm{CDH}^{79} \mathrm{BrF}$, $\mathrm{CDH}^{81} \mathrm{BrF}, \mathrm{CD}_{2}{ }^{79} \mathrm{BrF}$, and $\mathrm{CD}_{2}{ }^{81} \mathrm{BrF}$, thus enabling the determination of the spin-rotation constants of bromine for the first time. The experimental investigations have been supported by quantum-chemical computations and in particular the theoretical values for the bromine spin-rotation constants provided an essential support for recording and assigning the Lamb-dip spectra. Concerning the bromine quadrupole-coupling tensor, we note that relativistic corrections turned out to be essential for reliable predictions and that those are available with sufficient accuracy from a second-order direct perturbation theory (DPT2) treatment. 


\section{Acknowledgments}

Michael E. Harding (Mainz) is thanked for help in performing the calculations using the parallel version of ACESII. This work has been supported by 'PRIN 2005' funds (project "Trasferimenti di energia e di carica a livello molecolare") and by the University of Bologna (RFO funds) as well as in Mainz by the Deutsche Forschungsgemeinschaft and the Fonds der Chemischen Industrie. 


\section{Captions of the Tables}

Table 1. Comparison of experimental and theoretical ground-state rotational constants, centrifugal distortion constants, and bromine hyperfine parameters of $\mathrm{CH}_{2}{ }^{79} \mathrm{BrF}$ and $\mathrm{CH}_{2}{ }^{81} \mathrm{BrF}$.

Table 2. Comparison of experimental and theoretical ground-state rotational constants, centrifugal distortion constants, and bromine hyperfine parameters of $\mathrm{CDH}^{79} \mathrm{BrF}$ and $\mathrm{CDH}^{81} \mathrm{BrF}$.

Table 3. Comparison of experimental and theoretical ground-state rotational constants, centrifugal distortion constants, and bromine hyperfine parameters of $\mathrm{CD}_{2}{ }^{79} \mathrm{BrF}$ and $\mathrm{CD}_{2}{ }^{81} \mathrm{BrF}$.

Table 4. Individual contributions to the computed bromine quadrupole-coupling tensors (in $\mathrm{MHz}$ ) of the considered isotopologues of $\mathrm{CH}_{2} \mathrm{FBr}$. The non-relativistic values have been obtained at the $\operatorname{CCSD}(\mathrm{T}) / \mathrm{cc}-\mathrm{pCVQZ}$ level, the relativistic DPT2 corrections at the $\operatorname{CCSD}(\mathrm{T}) / \mathrm{cc}-\mathrm{pVQZ}$-unc level and the vibrational corrections at the MP2/cc-pCVTZ level.

Table 5. Comparison of experimental and theoretical bromine principal quadrupolecoupling tensor of $\mathrm{CH}_{2}{ }^{79} \mathrm{BrF}, \mathrm{CH}_{2}{ }^{81} \mathrm{BrF}, \mathrm{CHD}^{79} \mathrm{BrF}, \mathrm{CHD}^{81} \mathrm{BrF}, \mathrm{CD}_{2}{ }^{79} \mathrm{BrF}$ and $\mathrm{CD}_{2}{ }^{81} \mathrm{BrF}$. Values in $\mathrm{MHz}$.

Table 6. Quantum-chemically computed deuterium quadrupole-coupling constants. Values in $\mathrm{MHz}$.

Table 7. Quantum-chemically computed spin-rotation constants of fluorine and hydrogen. Values in $\mathrm{kHz}$.

\section{Captions of the Figures}

Figure 1. Portion of the Lamb-dip spectrum of the $\mathrm{CH}_{2} \mathrm{BrF}, \mathrm{CHDBrF}$ and $\mathrm{CD}_{2} \mathrm{BrF}$ mixture recorded in the 539.12-539.14 GHz frequency range. (a) Comparison between sub-Doppler (modulation depth $=45 \mathrm{kHz}$ ) and Doppler limited (modulation depth $=600 \mathrm{kHz}$ ) experimental spectra. In the insets, small portions of spectra of $\mathrm{CD}_{2}{ }^{81} \mathrm{BrF}$ are shown in details. (b) A $\Delta J=0$, with $J=64$, 
rotational transition of $\mathrm{CH}_{2}{ }^{79} \mathrm{BrF}$ is shown in detail and compared with the calculated spectrum.

Figure 2. Comparison between sub-Doppler (modulation depth $=30 \mathrm{kHz}$ ) and Doppler limited (modulation depth $=400 \mathrm{kHz}$ ) spectra of the $\Delta F=$ $0(F=49 / 2,51 / 2,53 / 2,55 / 2)$ resolved components of the $26_{6,20} \leftarrow 26_{5,21}$ and $26_{6,21} \leftarrow 26_{5,22}$ rotational transitions of $\mathrm{CH}_{2}{ }^{79} \mathrm{BrF}$.

Figure 3. Prediction of the splitting due to deuterium of the $2_{2,0} \leftarrow 1_{1,1}$ rotational transition of $\mathrm{CHD}^{79} \mathrm{BrF}$, where the quantum numbers $F_{1}$ and $F_{\text {tot }}$ come from the coupling scheme $F_{1}=J+I_{B r}$ and $F_{t o t}=F_{1}+I_{D}$, respectively: (a) modulation depth $=20 \mathrm{kHz}$, (b) modulation depth $=25 \mathrm{kHz}$. The comparison with experiment (c) is reported. In the experimental spectrum the Doppler profile is partially evident. 


\section{References}

[1] W. Gordy and R. L. Cook, Microwave molecular spectra, 3rd Edition, edited by A. Weissberger (Wiley, New York, 1984).

[2] G. Cazzoli, C. Puzzarini, A. Gambi, and J. Gauss, J. Chem. Phys. 125, 054313-1 (2006).

[3] F. Pawłowski, P. Jørgensen, J. Olsen, F. Hegelund, T. Helgaker, J. Gauss, K.L. Bak, and J.F. Stanton, J. Chem. Phys. 116, 6482 (2002).

[4] O. Baum, S. Esser, N. Gierse, S. Brünken, F. Lewen, J. Hahn, J. Gauss, S. Schlemmer, and T.F. Giesen, J. Mol. Struct. 795256 (2006).

[5] C. Puzzarini, S. Coriani, A. Rizzo, and J. Gauss, Chem. Phys. Lett. 409, 118 (2005).

[6] A. Rizzo, C. Puzzarini, S. Coriani, and J. Gauss, J. Chem. Phys. 124, 064302-1 (2005).

[7] V. Kellö and A. J. Sadlej, Int. J. Quantum Chem. 68, 159 (1998).

[8] M. Pernpointer and L. Visscher, J. Chem. Phys. 114, 10389 (2001).

[9] I. Malkin, O. L. Malkina, and V. G. Malkin, Chem. Phys. Lett. 361, 231 (2002).

[10] A, Rizzo, K. Ruud, and P. Norman, J. Mol. Struct. (Theochem) 633, 163 (2003).

[11] G. Cazzoli, C. Puzzarini, A. Baldacci, and A. Baldan, J. Mol. Spectrosc. 241, $112(2007)$.

[12] A. Baldacci, P. Stoppa, A. Pietropolli Charmet, S. Giorgianni, G. Cazzoli, C. Puzzarini, and R.W. Larsen, J. Phys. Chem. A 111, 7090 (2007).

[13] A. Baldacci, P. Stoppa, A. Pietropolli Charmet, S. Giorgianni, G. Cazzoli, L. Cludi, C. Puzzarini, and R.W. Larsen, J. Mol. Spectrosc. 246, 126 (2007).

[14] C. Puzzarini, G. Cazzoli, A. Baldacci, A. Baldan, C. Michauk, and J. Gauss, J. Chem. Phys. 127, 164302-1 (2007).

[15] R. Atkinson and W. P. L. Carter, Chem. Rev. 84, 437 (1984).

[16] R. Atkinson, Chem. Rev. 86, 69 (1986).

[17] R. Atkinson, Atmos. Environ. 24A, 1 (1990).

[18] Word Meteorological Organization (WMO), Scientific Assessment of Ozone Depletion: 2002. Global Ozone, Research and Monitoring Project. Report 47, Geneva, 2003.

[19] G. Cazzoli and L. Dore, J. Mol. Spectrosc. 143, 231 (1990).

[20] L. Dore, C. Degli Esposti, A. Mazzavillani, and G. Cazzoli, Chem. Phys. Lett. 300, 489 (1999). 
[21] G. Cazzoli and L. Dore, J. Mol. Spectrosc. 141, 49 (1990).

[22] G. Cazzoli, C. Puzzarini, and A.V. Lapinov, Astrophys. J. 592, L95 (2003).

[23] C. Puzzarini, L. Dore, and G. Cazzoli, J. Mol. Spectrosc. 217, 19 (2003).

[24] G. Cazzoli, L. Dore, L. Cludi, C. Puzzarini, and S. Beninati, J. Mol. Spectrosc. 215, 160 (2002).

[25] G. Cazzoli, L. Dore, C. Puzzarini, and S. Beninati, Phys. Chem. Chem. Phys. 4, 3575 (2002).

[26] E. A. C. Lucken, Nuclear Quadrupole Coupling Constants (Academic Press, London/New York, 1969).

[27] W. H. Flygare, J. Chem. Phys. 41, 793 (1964).

[28] W. H. Flygare, Chem. Rev. 74, 653 (1974).

[29] J. F. Stanton and J. Gauss, Int. Rev. Phys. Chem. 19, 61 (2000).

[30] J. Gauss and J. F. Stanton, J. Chem. Phys. 104, 257 (1996).

[31] J. Gauss and J. F. Stanton, Chem. Phys. Lett. 276, 70 (1997).

[32] M. Kállay and J. Gauss, J. Chem. Phys. 120, 6841 (2004).

[33] J. Gauss, K. Ruud, and T. Helgaker, J. Chem. Phys. 105, 2804 (1996).

[34] For recent reviews, see: R. J. Bartlett and J. F. Stanton, in Reviews in Computational Chemistry, Vol. 5, edited by K. B. Lipkowitz, D. B. Boyd (VCH Publisher, New York, 1994); T. J. Lee and G. E. Scuseria, in Quantum Mechanical Electronic Structure Calculations with Chemical Accuracy, edited by S. R. Langhoff (Kluwer Academic Publisher, Dordrecht, 1995); R. J. Bartlett, in Modern Electronic Structure Theory, edited by D. R. Yarkony (World Scientific, Singapore, 1995); J. Gauss, in Encyclopedia of Computational Chemistry, edited by P. v. R. Schleyer, N. L. Allinger, T. Clark, J. Gasteiger, P. A. Kollmann, H. F. Schaefer, and P. R. Schreiner (Wiley, New York, 1998).

[35] K. Raghavachari, G. W. Trucks, J. A. Pople, and M. Head-Gordon, Chem. Phys. Lett. 157, 479 (1989).

[36] K. Peterson and T. H. Dunning, Jr., J. Chem. Phys. 117, 10548 (2002).

[37] N. J. DeYonker, K. A. Peterson, and A. K. Wilson, J. Phys. Chem. A 111, 11383 (2007).

[38] W. Kutzelnigg, in Relativistic Electronic Structure Theory Part I. Fundamentals, edited by P. Schwerdtfeger (Elsevier, Amsterdam, 2002), p. 664.

[39] W. Klopper, J. Comp. Chem. 18, 20 (1997).

[40] W. Klopper, S. Coriani, T. Helgaker, and P. Jørgensen, J. Phys. B 37, 3753 (2004). 
[41] S. Stopkowicz, diploma thesis, Universität Mainz, 2008; S. Stopkowicz and J. Gauss, J. Chem. Phys., to be submitted.

[42] P. Pyykkö, Z. Naturforsch. 47A, 189 (1991).

[43] J. Bieron, P. Pykkö, D. Sundholm, V. Kellö, and A. J. Sadlej, Phys. Rev. A 64, 052507 (2001).

[44] A. A. Auer, J. Gauss, and J. F. Stanton, J. Chem. Phys. 118, 10407 (2003).

[45] C. Møller and M. S. Plesset, Phys. Rev. 46, 618 (1934).

[46] Best estimated equilibrium structure: $\mathrm{C}-\mathrm{F}=1.3578 \AA, \mathrm{C}-\mathrm{Br}=1.9256 \AA, \mathrm{C}-\mathrm{H}$ $=1.0833 \AA, \angle \mathrm{FCBr}=110.14$ degree, $\angle \mathrm{HCF}=109.51$ degree, $\angle \mathrm{HCBr}=107.27$ degree, $\angle \mathrm{HCH}=113.09$ degree.

[47] ACESII (Mainz-Austin-Budapest version), a quantum-chemical program package for high-level calculations of energies and properties by J. F. Stanton, J. Gauss, J. D. Watts et al., see http://www.aces2.de.

[48] M. E. Harding, T. Metzroth, A. A. Auer, and J. Gauss, J. Chem. Theor. Comp. 4, 64 (2008).

[49] AABS package (available from http://www.ifpan.edu.pl/ kisiel/aabs/aabs. htm): Z. Kisiel, L. Pszczolkowski, I. R.Medvedev, M. Winnewisser, F. C. De Lucia, C. E. Herbst, J. Mol. Spectrosc. 233, 231 (2005).

[50] H. M. Pickett, J. Mol. Spectrosc. 148, 371 (1991).

[51] J. K. G. Watson, in Vibrational Spectra and Structure, Vol. 6, edited by J.R. Durig (Elsevier, New York/Amsterdam, 1977). 
Table 1

Comparison of experimental and theoretical ground-state rotational constants, centrifugal distortion constants, and bromine hyperfine parameters of $\mathrm{CH}_{2}{ }^{79} \mathrm{BrF}$ and $\mathrm{CH}_{2}{ }^{81} \mathrm{BrF}$.

\begin{tabular}{|c|c|c|c|c|c|c|c|}
\hline & & \multicolumn{3}{|c|}{$\mathrm{CH}_{2}{ }^{79} \mathrm{BrF}$} & \multicolumn{3}{|c|}{$\mathrm{CH}_{2}{ }^{81} \mathrm{BrF}$} \\
\hline & & Ref.[12] & Exp. & Calc. $^{a}$ & Ref.[13] & Exp. & Calc. $^{a}$ \\
\hline$A_{0}$ & $(\mathrm{MHz})$ & $39852.97118(62)$ & $39852.97088(46)$ & 39973.0 & $39841.92452(64)$ & $39841.92220(19)$ & 39961.9 \\
\hline$B_{0}$ & $(\mathrm{MHz})$ & $3772.957168(69)$ & $3772.957189(44)$ & 3780.6 & $3747.116199(75)$ & $3747.116117(29)$ & 3754.7 \\
\hline$C_{0}$ & $(\mathrm{MHz})$ & $3523.953868(71)$ & $3523.953911(51)$ & 3531.1 & $3501.314903(76)$ & $3501.314830(30)$ & 3508.4 \\
\hline$\Delta_{J}$ & $(\mathrm{kHz})$ & $1.787928(51)$ & $1.787911(33)$ & 1.70 & $1.764907(42)$ & $1.764810(13)$ & 16.82 \\
\hline$\Delta_{J K}$ & $(\mathrm{kHz})$ & $-24.7916(13)$ & $-24.79359(34)$ & -24.37 & $-24.6418(10)$ & $-24.638763(74)$ & -24.22 \\
\hline$\Delta_{K}$ & $(\mathrm{kHz})$ & $538.660(28)$ & $538.682(19)$ & 541.10 & $538.181(23)$ & $538.0623(52)$ & 540.48 \\
\hline$\delta_{J}$ & $(\mathrm{kHz})$ & $0.183517(16)$ & $0.183502(15)$ & 0.17 & $0.179929(53)$ & $0.1799878(58)$ & 0.17 \\
\hline$\delta_{K}$ & $(\mathrm{kHz})$ & $8.0991(70)$ & $8.1027(59)$ & 7.43 & $8.0542(35)$ & $8.0143(29)$ & 7.35 \\
\hline$\Phi_{J}$ & $(\mathrm{~Hz})$ & $0.0009003(72)$ & $0.0008871(70)$ & & $0.0008890(59)$ & $0.0008614(29)$ & \\
\hline$\Phi_{J K}$ & $(\mathrm{~Hz})$ & $0.01077(22)$ & $0.0218(22)$ & & $0.01019(20)$ & $0.02377(82)$ & \\
\hline$\Phi_{K J}$ & $(\mathrm{~Hz})$ & $-1.939(13)$ & $-2.009(12)$ & & $-1.9734(96)$ & $-2.0168(39)$ & \\
\hline$\Phi_{K}$ & $(\mathrm{~Hz})$ & $25.12(57)$ & $25.70(28)$ & & $27.40(42)$ & $26.000(38)$ & \\
\hline$\phi_{J}$ & $(\mathrm{~Hz})$ & $0.0002630(29)$ & $0.0002735(45)$ & & $0.0002400(12)$ & $0.0002708(18)$ & \\
\hline$\phi_{J K}$ & $(\mathrm{~Hz})$ & $0.0248(14)$ & $0.0129(28)$ & & $0.0311(10)$ & $0.0091(13)$ & \\
\hline$\phi_{K}$ & $(\mathrm{~Hz})$ & & $2.04(41)$ & & & $2.44(15)$ & \\
\hline$\chi_{a a}$ & $(\mathrm{MHz})$ & $443.531(24)$ & $443.431(8)$ & 449.70 & $370.724(28)$ & $370.6497(59)$ & 375.84 \\
\hline$\chi_{b b}-\chi_{c c}$ & $(\mathrm{MHz})$ & $153.571(34)$ & $153.566(26)$ & 156.38 & $128.086(39)$ & $127.966(22)$ & 129.98 \\
\hline$\left|\chi_{a b}\right|$ & $(\mathrm{MHz})$ & $278.63(54)$ & $278.56(19)$ & 284.40 & $232.44(13)$ & $232.657(46)$ & 236.96 \\
\hline$\chi_{a a}^{J}$ & $(\mathrm{MHz})$ & & $0.000160(45)$ & & $0.00060(9)$ & $0.000127(35)$ & \\
\hline$\chi_{a a}^{K}$ & $(\mathrm{MHz})$ & $-0.00839(66)$ & $-0.00441(18)$ & & $-0.0045(8)$ & $-0.00361(13)$ & \\
\hline$C_{a a}$ & $(\mathrm{kHz})$ & & $15.63(24)$ & $15.34(15.64)$ & & $16.59(16)$ & 15.40 \\
\hline$C_{b b}$ & $(\mathrm{kHz})$ & & $4.97(27)$ & $5.24(5.30)$ & & $4.95(16)$ & 5.22 \\
\hline$C_{c c}$ & $(\mathrm{kHz})$ & & $8.43(28)$ & $8.92(9.04)$ & & $9.38(15)$ & 8.88 \\
\hline$C_{a b}$ & $(\mathrm{kHz})$ & & & $11.09(10.74)$ & & & 11.13 \\
\hline$C_{b a}$ & $(\mathrm{kHz})$ & & & $1.60(1.60)$ & & & 1.59 \\
\hline$\sigma^{b}$ & $(\mathrm{kHz})$ & 22.8 & 12.5 & & 22.7 & 10.5 & \\
\hline
\end{tabular}

a Spectroscopic parameters from the (fc) CCSD(T)/cc-pVTZ force field [14]. Rotational constants are based on the best estimated ab initio geometry from Table V of Ref. [14] and vibration-rotation interaction constants from the above-mentioned force field (Ref. [14]). Quadrupole coupling constants computed at the $\operatorname{CCSD}(\mathrm{T}) / \mathrm{cc}-\mathrm{pCVQZ}$ level and corrected for relativistic effects computed at the $\operatorname{CCSD}(\mathrm{T}) / \mathrm{cc}-$ pVQZ-unc level using DPT2 and vibrational corrections evaluated at the MP2/cc-pCVTZ level: see text. Spin-rotation evaluated at the MP2/cc-pCVTZ level: see text. Spin-rotation constants computed at the $\operatorname{CCSD}(\mathrm{T}) / \mathrm{cc}-\mathrm{pCVTZ}$ level and corrected for vibrational corrections evaluated at the MP2/cc-pCVTZ level: see text. For $\mathrm{CH}_{2}{ }^{79} \mathrm{BrF}$ in parentheses values computed at the $\mathrm{CCSD}(\mathrm{T}) / \mathrm{cc}-\mathrm{pCVQZ}$ level (also corrected for vibrational effects) are given.

${ }^{b}$ Standard deviation of the fit. 
Table 2

Comparison of experimental and theoretical ground-state rotational constants, centrifugal distortion constants, and bromine hyperfine parameters of $\mathrm{CDH}^{79} \mathrm{BrF}$ and $\mathrm{CDH}^{81} \mathrm{BrF}$.

\begin{tabular}{|c|c|c|c|c|c|c|c|}
\hline & & \multicolumn{3}{|c|}{$\mathrm{CDH}^{79} \mathrm{BrF}$} & \multicolumn{3}{|c|}{$\mathrm{CDH}^{81} \mathrm{BrF}$} \\
\hline & & Ref.[14] & Exp. & Calc. $^{a}$ & Ref.[14] & Exp. & Calc. $^{a}$ \\
\hline$A_{0}$ & $(\mathrm{MHz})$ & $33679.3876(19)$ & $33679.38682(33)$ & 33795.7 & $33670.63098(51)$ & $33670.63189(26)$ & 33786.9 \\
\hline$B_{0}$ & $(\mathrm{MHz})$ & $3711.04022(16)$ & $3711.040208(49)$ & 3718.9 & $3685.18414(12)$ & $3685.183896(49)$ & 3693.0 \\
\hline$C_{0}$ & $(\mathrm{MHz})$ & $3447.68974(17)$ & $3447.689756(51)$ & 3455.0 & $3425.27136(11)$ & $3425.271166(53)$ & 3432.6 \\
\hline$\Delta_{J}$ & $(\mathrm{kHz})$ & $1.66264(11)$ & $1.662641(38)$ & 1.59 & $1.640893(64)$ & $1.640779(31)$ & 1.57 \\
\hline$\Delta_{J K}$ & $(\mathrm{kHz})$ & $-16.64703(95)$ & $-16.64713(32)$ & -16.32 & $-16.5428(11)$ & $-16.54335(31)$ & -16.22 \\
\hline$\Delta_{K}$ & $(\mathrm{kHz})$ & $324.743(49)$ & $324.7219(88)$ & 325.24 & $324.293(11)$ & $324.3142(71)$ & 324.86 \\
\hline$\delta_{J}$ & $(\mathrm{kHz})$ & $0.180907(63)$ & $0.180902(23)$ & 0.17 & $0.177358(47)$ & $0.177341(18)$ & 0.17 \\
\hline$\delta_{K}$ & $(\mathrm{kHz})$ & $6.962(22)$ & $6.9624(82)$ & 6.47 & $6.879(16)$ & $6.8774(69)$ & 6.39 \\
\hline$\Phi_{J}$ & $(\mathrm{~Hz})$ & $0.000772(27)$ & $0.000772(10)$ & & $0.000741(14)$ & $0.0007214(74)$ & \\
\hline$\Phi_{J K}$ & $(\mathrm{~Hz})$ & $0.01029(36)$ & $0.01031(12)$ & & $0.01065(39)$ & $0.01033(14)$ & \\
\hline$\Phi_{K J}$ & $(\mathrm{~Hz})$ & $-1.093(13)$ & $-1.0942(51)$ & & $-1.0560(87)$ & $-1.0586(49)$ & \\
\hline$\Phi_{K}$ & $(\mathrm{~Hz})$ & $11.72(39)$ & $11.554(84)$ & & $11.12(11)$ & $11.231(70)$ & \\
\hline$\phi_{J}$ & $(\mathrm{~Hz})$ & $0.000257(19)$ & $0.0002561(72)$ & & $0.000246(13)$ & $0.0002420(51)$ & \\
\hline$\phi_{J K}$ & $(\mathrm{~Hz})$ & $0.0151(93)$ & $0.0153(34)$ & & $0.0104(73)$ & $0.0086(36)$ & \\
\hline$\chi_{a a}$ & $(\mathrm{MHz})$ & $450.327(43)$ & $450.310(11)$ & 456.60 & $376.327(22)$ & $376.383(11)$ & 381.71 \\
\hline$\chi_{b b-}-\chi_{c c}$ & $(\mathrm{MHz})$ & $141.72(11)$ & $141.818(28)$ & 144.27 & $118.284(32)$ & $118.282(22)$ & 120.30 \\
\hline$\left|\chi_{a b}\right|$ & $(\mathrm{MHz})$ & $269.12(50)$ & $269.77(33)$ & 273.89 & $224.68(33)$ & $223.65(36)$ & 228.58 \\
\hline$\chi_{b c}$ & $(\mathrm{MHz})$ & {$[-18.54]^{b}$} & $-17.75(89)$ & -18.62 & {$[-15.38]^{b}$} & $-14.69(91)$ & -15.46 \\
\hline$\chi_{a c}$ & $(\mathrm{MHz})$ & {$[36.78]^{b}$} & $28.7(33)$ & 37.58 & {$[30.67]^{b}$} & $33.5(22)$ & 30.77 \\
\hline$\chi_{a a}^{J}$ & $(\mathrm{MHz})$ & $-0.00085(23)$ & $-0.000647(60)$ & & & $-0.000365(64)$ & \\
\hline$\chi_{a a}^{K}$ & $(\mathrm{MHz})$ & $-0.0051(7)$ & $-0.00424(17)$ & & $-0.0026(9)$ & $-0.00315(18)$ & \\
\hline$C_{a a}$ & $(\mathrm{kHz})$ & & $12.72(41)$ & 12.59 & & $15.02(44)$ & 13.55 \\
\hline$C_{b b}$ & $(\mathrm{kHz})$ & & $4.64(47)$ & 5.22 & & $5.99(40)$ & 5.59 \\
\hline$C_{c c}$ & $(\mathrm{kHz})$ & & $8.42(46)$ & 8.65 & & $9.78(54)$ & 9.27 \\
\hline$C_{a b}$ & $(\mathrm{kHz})$ & & & 8.71 & & & 9.37 \\
\hline$C_{b a}$ & $(\mathrm{kHz})$ & & & 1.50 & & & 1.60 \\
\hline$C_{a c}$ & $(\mathrm{kHz})$ & & & 1.65 & & & 1.78 \\
\hline$C_{c a}$ & $(\mathrm{kHz})$ & & & 0.23 & & & 0.24 \\
\hline$C_{b c}$ & $(\mathrm{kHz})$ & & & -0.47 & & & -0.51 \\
\hline$C_{c b}$ & $(\mathrm{kHz})$ & & & -0.44 & & & -0.47 \\
\hline$\sigma^{c}$ & $(\mathrm{kHz})$ & 22.4 & 12.3 & & 18.9 & 11.5 & \\
\hline
\end{tabular}

a Spectroscopic parameters from the (fc) CCSD(T)/cc-pVTZ force field [14]. Rotational constants are based on the best estimated ab initio geometry from Eq.(4) of Ref. [14] and vibration-rotation interaction constants from the above-mentioned force field (Ref. [14]). Quadrupole-coupling constants computed at the CCSD(T)/cc-pCVQZ level and corrected for relativistic effects computed at the CCSD(T)/cc-pVQZunc level using DPT2 and vibrational corrections evaluated at the MP2/cc-pCVTZ level: see text. Spinrotation constants computed at the $\operatorname{CCSD}(\mathrm{T}) / \mathrm{cc}-\mathrm{pCVTZ}$ level and corrected for vibrational corrections evaluated at the MP2/cc-pCVTZ level: see text.

$b$ Fixed at the computed values.

$c$ Standard deviation of the fit. 


\section{Table 3}

Comparison of experimental and theoretical ground-state rotational constants, centrifugal distortion constants, and bromine hyperfine parameters of $\mathrm{CD}_{2}{ }^{79} \mathrm{BrF}$ and $\mathrm{CD}_{2}{ }^{81} \mathrm{BrF}$.

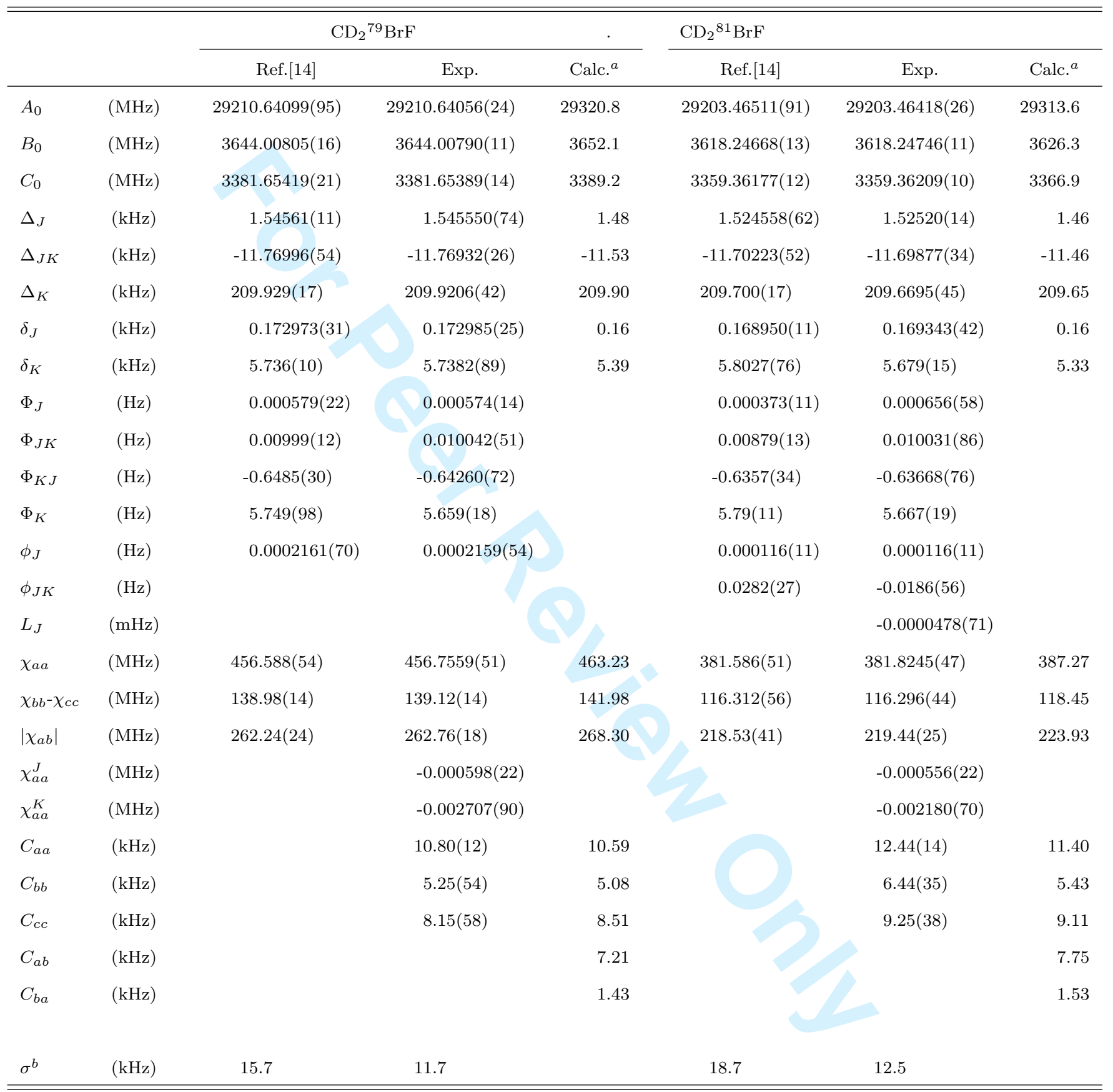

a Spectroscopic parameters from the (fc) CCSD(T)/cc-pVTZ force field [14]. Rotational constants are based on the best estimated ab initio geometry from Eq.(4) of Ref. [14] and vibration-rotation interaction constants from the above-mentioned force field (Ref. [14]). Quadrupole-coupling constants computed at the CCSD(T)/cc-pCVQZ level and corrected for relativistic effects computed at the CCSD(T)/cc-pVQZunc level using DPT2 and vibrational corrections evaluated at the MP2/cc-pCVTZ level: see text. Spinrotation constants computed at the $\operatorname{CCSD}(\mathrm{T}) / \mathrm{cc}-\mathrm{pCVTZ}$ level and corrected for vibrational corrections evaluated at the MP2/cc-pCVTZ level: see text.

$b$ Standard deviation of the fit. 
Table 4

Individual contributions to the computed bromine quadrupole-coupling tensors (in $\mathrm{MHz}$ ) of the considered isotopologues of $\mathrm{CH}_{2} \mathrm{FBr}$. The non-relativistic values have been obtained at the CCSD $(\mathrm{T}) / \mathrm{cc}-\mathrm{pCVQZ}$ level, the relativistic DPT2 corrections at the CCSD(T)/cc-pVQZ-unc level and the vibrational corrections at the MP2/ccpCVTZ level.

\begin{tabular}{|c|c|c|c|c|}
\hline & Non-rel. & DPT2 & Vib. Corr. & Total \\
\hline \multicolumn{5}{|c|}{$\mathrm{CH}_{2}{ }^{79} \mathrm{BrF}$} \\
\hline$\chi_{a a}$ & 421.84 & 27.47 & 0.39 & 449.70 \\
\hline$\chi_{b b}$ & -138.68 & -9.06 & 1.08 & -146.67 \\
\hline$\chi_{c c}$ & -283.17 & -18.41 & -1.47 & -303.05 \\
\hline$\chi_{a b}$ & -264.74 & -17.48 & -2.18 & -284.40 \\
\hline \multicolumn{5}{|c|}{$\mathrm{CH}_{2}{ }^{81} \mathrm{BrF}$} \\
\hline$\chi_{a a}$ & 352.64 & 22.97 & 0.24 & 375.85 \\
\hline$\chi_{b b}$ & -116.06 & -7.59 & 0.72 & -122.93 \\
\hline$\chi_{c c}$ & -236.57 & -15.38 & -0.96 & -252.91 \\
\hline$\chi_{a b}$ & -220.96 & -14.59 & -1.41 & -236.96 \\
\hline \multicolumn{5}{|c|}{$\mathrm{CHD}^{79} \mathrm{BrF}$} \\
\hline$\chi_{a a}$ & 428.41 & 27.60 & 0.28 & 456.60 \\
\hline$\chi_{b b}$ & -147.40 & -9.64 & 0.87 & -156.17 \\
\hline$\chi_{c c}$ & -281.02 & -18.27 & -1.15 & -300.44 \\
\hline$\chi_{a b}$ & -255.35 & -16.87 & -1.68 & -273.89 \\
\hline$\chi_{a c}$ & 34.62 & 2.24 & 0.72 & 37.58 \\
\hline$\chi_{b c}$ & -17.07 & -1.10 & -0.45 & -18.62 \\
\hline \multicolumn{5}{|c|}{$\mathrm{CHD}^{81} \mathrm{BrF}$} \\
\hline$\chi_{a a}$ & 358.14 & 23.33 & 0.24 & 381.71 \\
\hline$\chi_{b b}$ & -123.36 & -8.07 & 0.72 & -130.71 \\
\hline$\chi_{c c}$ & -234.78 & -15.26 & -0.96 & -251.00 \\
\hline$\chi_{a b}$ & -213.10 & -14.07 & -1.41 & -228.58 \\
\hline$\chi_{a c}$ & 28.30 & 1.87 & 0.60 & 30.77 \\
\hline$\chi_{b c}$ & -14.16 & -0.92 & -0.38 & -15.46 \\
\hline \multicolumn{5}{|c|}{$\mathrm{CD}_{2}{ }^{79} \mathrm{BrF}$} \\
\hline$\chi_{a a}$ & 434.71 & 28.32 & 0.20 & 463.23 \\
\hline$\chi_{b b}$ & -151.54 & -9.91 & 0.83 & -160.62 \\
\hline$\chi_{c c}$ & -283.17 & -18.41 & -1.03 & -302.61 \\
\hline$\chi_{a b}$ & -250.42 & -16.54 & -1.34 & -268.30 \\
\hline \multicolumn{5}{|c|}{$\mathrm{CD}_{2}{ }^{81} \mathrm{BrF}$} \\
\hline$\chi_{a a}$ & 363.41 & 23.67 & 0.18 & 387.27 \\
\hline$\chi_{b b}$ & -126.84 & -8.30 & 0.73 & -134.41 \\
\hline$\chi_{c c}$ & -236.57 & -15.38 & -0.91 & -252.86 \\
\hline$\chi_{a b}$ & -208.95 & -13.80 & -1.18 & -223.93 \\
\hline
\end{tabular}


Table 5

Comparison of experimental and theoretical bromine quadrupole-coupling tensor of $\mathrm{CH}_{2}{ }^{79} \mathrm{BrF}, \mathrm{CH}_{2}{ }^{81} \mathrm{BrF}, \mathrm{CHD}{ }^{79} \mathrm{BrF}, \mathrm{CHD}^{81} \mathrm{BrF}, \mathrm{CH}_{2}{ }^{79} \mathrm{BrF}$ and $\mathrm{CH}_{2}{ }^{81} \mathrm{BrF}$ in their principal axis system.

\begin{tabular}{|c|c|c|c|c|c|}
\hline & & Exp. & Calc. $^{a}$ & Exp. & Calc. $^{a}$ \\
\hline & & \multicolumn{2}{|c|}{$\mathrm{CH}_{2}{ }^{79} \mathrm{BrF}$} & \multicolumn{2}{|c|}{$\mathrm{CH}_{2}{ }^{81} \mathrm{BrF}$} \\
\hline$\chi_{z z}$ & $(\mathrm{MHz})$ & 554.389 & 563.59 & 463.244 & 470.47 \\
\hline$\chi_{x x}$ & $(\mathrm{MHz})$ & -255.891 & -260.54 & -213.936 & -217.56 \\
\hline$\chi_{y y}=\chi_{c c}{ }^{a}$ & $(\mathrm{MHz})$ & -298.499 & -303.05 & -249.308 & -252.91 \\
\hline & & \multicolumn{2}{|c|}{$\mathrm{CHD}^{79} \mathrm{BrF}$} & \multicolumn{2}{|c|}{$\mathrm{CHD}^{81} \mathrm{BrF}$} \\
\hline$\chi_{z z}$ & $(\mathrm{MHz})$ & 554.475 & 563.20 & 463.014 & 470.48 \\
\hline$\chi_{x x}$ & $(\mathrm{MHz})$ & -256.140 & -260.36 & -213.705 & -217.51 \\
\hline \multirow[t]{2}{*}{$\chi_{y y}$} & $(\mathrm{MHz})$ & -298.335 & -302.84 & -249.309 & -252.97 \\
\hline & & \multicolumn{2}{|c|}{$\mathrm{CD}_{2}{ }^{79} \mathrm{BrF}$} & \multicolumn{2}{|c|}{$\mathrm{CD}_{2}{ }^{81} \mathrm{BrF}$} \\
\hline$\chi_{z z}$ & $(\mathrm{MHz})$ & 553.661 & 562.74 & 462.693 & 470.21 \\
\hline$\chi_{x x}$ & $(\mathrm{MHz})$ & -255.723 & -260.13 & -213.633 & -217.35 \\
\hline$\chi_{y y}=\chi_{c c}{ }^{a}$ & $(\mathrm{MHz})$ & -297.950 & -302.61 & -249.060 & -252.86 \\
\hline
\end{tabular}

a This equality is due to the $C_{s}$ symmetry, where the inertial $c$ axis is that perpendicular to the symmetry plane $a b$. 
Table 6

Quantum-chemically computed deuterium quadrupole-coupling constants ${ }^{a}$. Values in $\mathrm{MHz}$.

\begin{tabular}{|c|c|c|c|c|}
\hline & $\mathrm{CHD}^{79} \mathrm{BrF}$ & $\mathrm{CHD}^{81} \mathrm{BrF}$ & $\mathrm{CD}_{2}{ }^{79} \mathrm{BrF}$ & $\mathrm{CD}_{2}{ }^{81} \mathrm{BrF}$ \\
\hline$\chi_{a a}$ & -0.084 & -0.083 & -0.084 & -0.084 \\
\hline$\chi_{b b}-\chi_{c c}$ & -0.052 & -0.052 & -0.027 & -0.027 \\
\hline$\chi_{a b}$ & -0.016 & -0.016 & -0.013 & -0.013 \\
\hline$\chi_{a c}$ & -0.028 & -0.028 & \pm 0.029 & \pm 0.029 \\
\hline$\chi_{b c}$ & 0.131 & 0.131 & \pm 0.122 & \pm 0.122 \\
\hline
\end{tabular}

40

41

43

44

45

46

47

48

49

50

51 
Table 7

Quantum-chemically computed spin-rotation constants of fluorine and hydrogen ${ }^{a}$. Values in $\mathrm{kHz}$.

\begin{tabular}{|c|c|c|c|c|c|c|}
\hline & $\mathrm{CH}_{2}{ }^{79} \mathrm{BrF}$ & $\mathrm{CH}_{2}{ }^{81} \mathrm{BrF}$ & $\mathrm{CHD}^{79} \mathrm{BrF}$ & $\mathrm{CHD}^{81} \mathrm{BrF}$ & $\mathrm{CD}_{2}{ }^{79} \mathrm{BrF}$ & $\mathrm{CD}_{2}{ }^{81} \mathrm{BrF}$ \\
\hline \multicolumn{7}{|l|}{$\mathrm{F}$} \\
\hline$C_{a a}$ & $-4.78(-4.56)$ & -4.77 & -3.63 & -3.62 & -2.80 & -2.80 \\
\hline$C_{b b}$ & $-2.55(-2.58)$ & -2.54 & -2.54 & -2.52 & -2.50 & -2.48 \\
\hline$C_{c c}$ & $-2.80(-2.91)$ & -2.78 & -2.72 & -2.70 & -2.66 & -2.65 \\
\hline$C_{a b}$ & $-13.01(-13.41)$ & -12.99 & -10.71 & -10.69 & -9.18 & -9.17 \\
\hline$C_{b a}$ & $-0.85(-0.87)$ & -0.85 & -0.81 & -0.80 & -0.78 & -0.77 \\
\hline$C_{a c}$ & & & 1.31 & 1.31 & & \\
\hline$C_{c a}$ & & & 0.10 & 0.09 & & \\
\hline$C_{b c}$ & & & -0.06 & -0.06 & & \\
\hline$C_{c b}$ & & & -0.06 & -0.06 & & \\
\hline \multicolumn{7}{|l|}{$\mathrm{H}$} \\
\hline$C_{a a}$ & $3.95(3.91)$ & 3.95 & 3.34 & 3.34 & & \\
\hline$C_{b b}$ & $0.19(0.19)$ & 0.19 & 0.24 & 0.24 & & \\
\hline$C_{c c}$ & $-0.12(-0.12)$ & -0.12 & -0.16 & -0.16 & & \\
\hline$C_{a b}$ & $0.60(0.60)$ & 0.60 & 0.43 & 0.43 & & \\
\hline$C_{b a}$ & $0.03(0.03)$ & 0.03 & 0.03 & 0.03 & & \\
\hline$C_{a c}$ & $\pm 0.79( \pm 0.79)$ & \pm 0.79 & -0.70 & -0.70 & & \\
\hline$C_{c a}$ & $\pm 0.05( \pm 0.05)$ & \pm 0.05 & -0.05 & -0.05 & & \\
\hline$C_{b c}$ & $\pm 0.22( \pm 0.22)$ & \pm 0.22 & 0.18 & 0.18 & & \\
\hline$C_{c b}$ & $\pm 0.27( \pm 0.27)$ & \pm 0.27 & 0.23 & 0.23 & & \\
\hline
\end{tabular}

a Computed at the CCSD $(\mathrm{T}) / \mathrm{cc}-\mathrm{pCVTZ}$ level and corrected for vibrational corrections evaluated at the MP2/cc-pCVTZ level: see text. For $\mathrm{CH}_{2}{ }^{79} \mathrm{BrF}$ in parentheses values computed at the CCSD(T)/ccpCVQZ level (also corrected for vibrational effects) are given. 

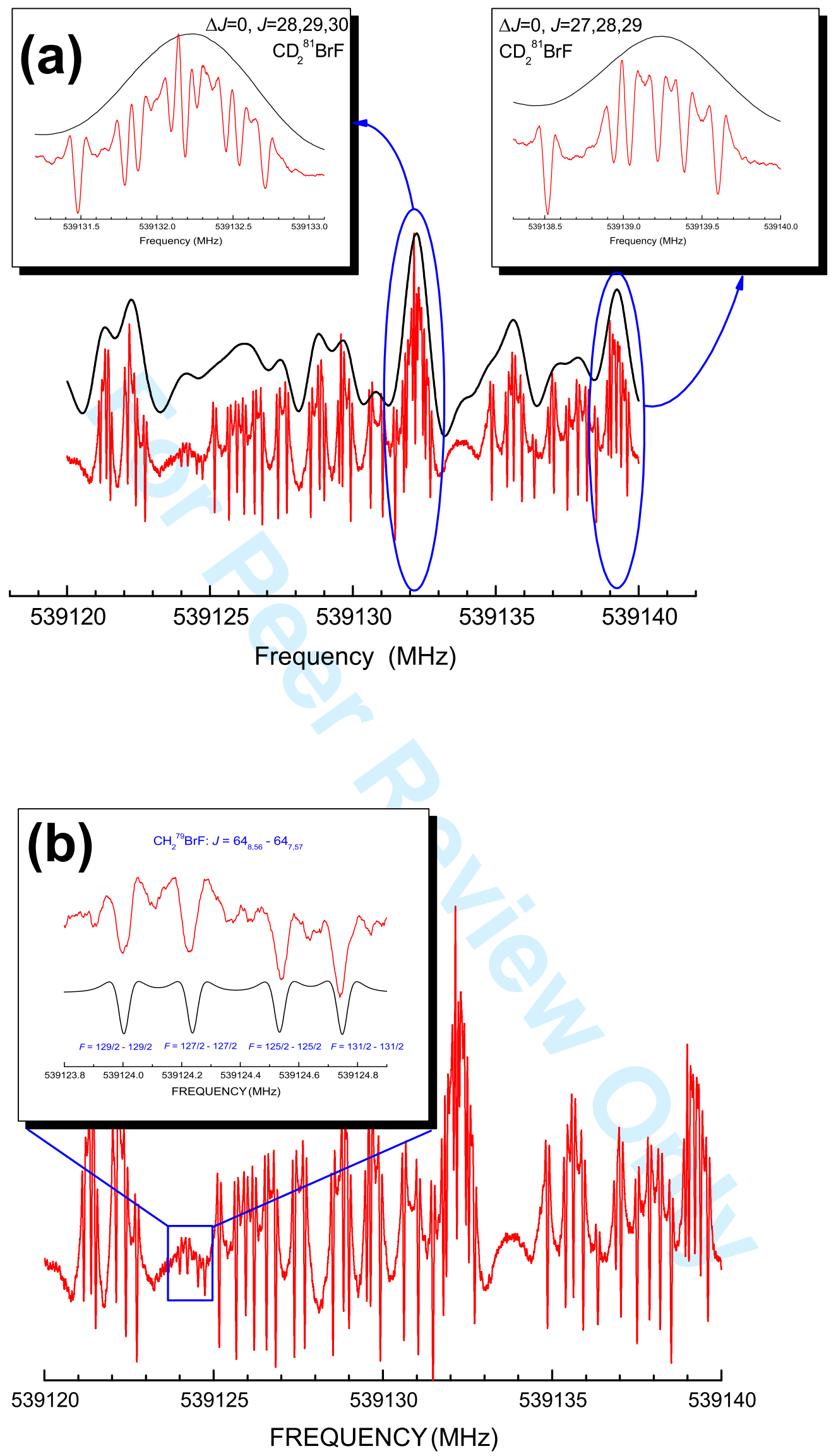

Fig. 1. Portion of the Lamb-dip spectrum of the $\mathrm{CH}_{2} \mathrm{BrF}, \mathrm{CHDBrF}$ and $\mathrm{CD}_{2} \mathrm{BrF}$ mixture recorded in the 539.12-539.14 GHz frequency range. (a) Comparison between sub-Doppler (modulation depth $=45 \mathrm{kHz}$ ) and Doppler limited (modulation depth $=600 \mathrm{kHz}$ ) experimental spectra. In the insets, small portions of spectra of $\mathrm{CD}_{2}{ }^{81} \mathrm{BrF}$ are shown in details. (b) A $\Delta J=0$, with $J=64$, rotational transition of $\mathrm{CH}_{2}{ }^{79} \mathrm{BrF}$ is shown in detail and compared with the calculated spectrum. 


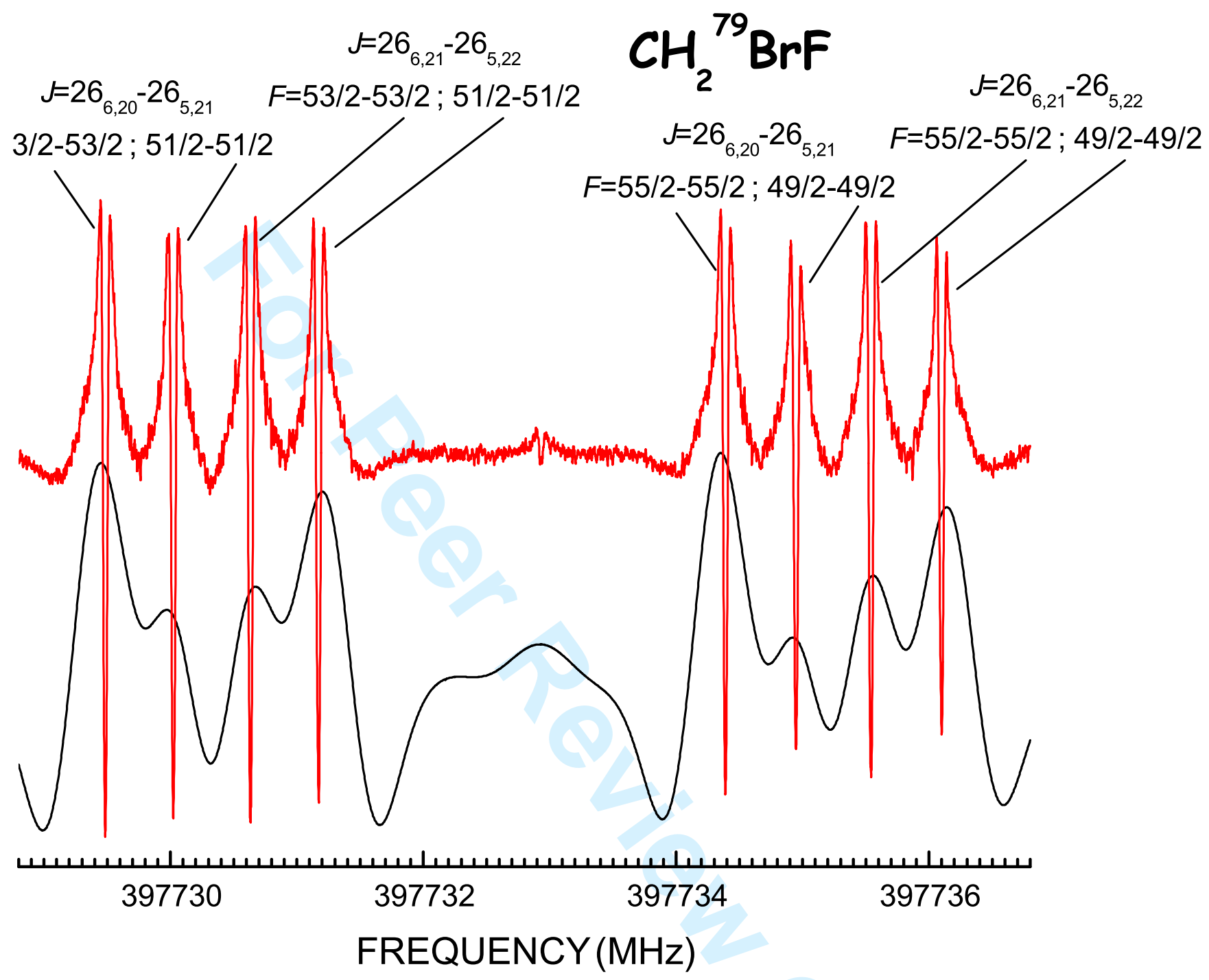

Fig. 2. Comparison between sub-Doppler (modulation depth $=30 \mathrm{kHz}$ ) and Doppler limited (modulation depth $=400 \mathrm{kHz}$ ) spectra of the $\Delta F=0$ $(F=49 / 2,51 / 2,53 / 2,55 / 2)$ resolved components of the $26_{6,20} \leftarrow 26_{5,21}$ and $26_{6,21}$ $\leftarrow 26_{5,22}$ rotational transitions of $\mathrm{CH}_{2}{ }^{79} \mathrm{BrF}$. 


\section{$\mathrm{CHD}^{79} \mathrm{BrF}: J=2_{2,0}-1_{1,1} ; F_{1}=7 / 2-5 / 2$}

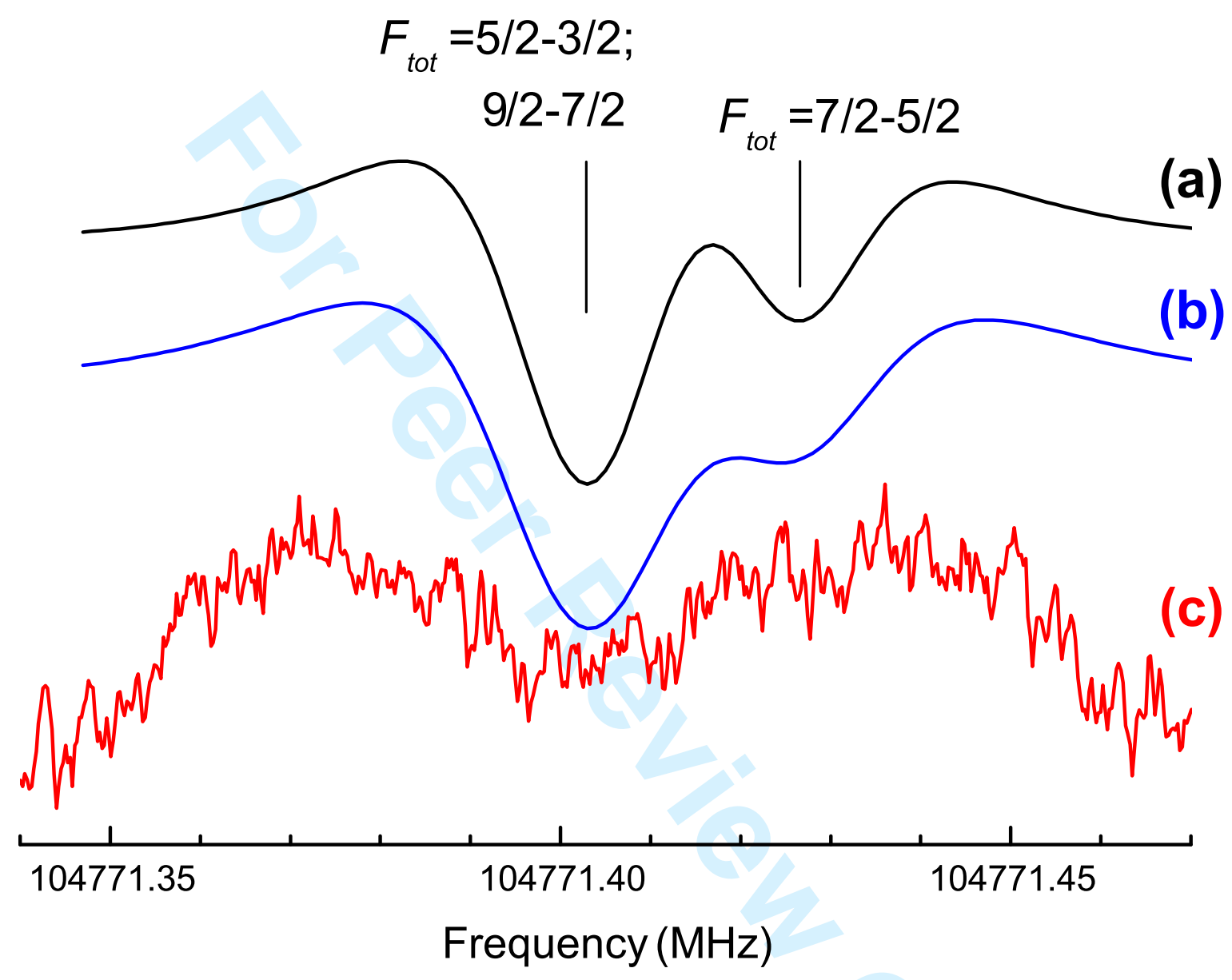

Fig. 3. Prediction of the splitting due to deuterium of the $2_{2,0} \leftarrow 1_{1,1}$ rotational transition of $\mathrm{CHD}^{79} \mathrm{BrF}$, where the quantum numbers $F_{1}$ and $F_{\text {tot }}$ come from the coupling scheme $F_{1}=J+I_{B r}$ and $F_{t o t}=F_{1}+I_{D}$, respectively: (a) modulation depth $=20 \mathrm{kHz}$, (b) modulation depth $=25 \mathrm{kHz}$. The comparison with experiment (c) is reported. In the experimental spectrum the Doppler profile is partially evident. 
2

3

4

5

6

7

8

9

10

11

12

13

14

15

16

17

18

19

20

21

22

23

24

25

26

27

28

29

30

31

32

33

34

35

36

37

38

39

40

41

42

43

44

45

46

47

48

49

50

51

52

53

54

55

56

57

58

59

60

URL: http://mc.manuscriptemiral.com/tandf/tmph 


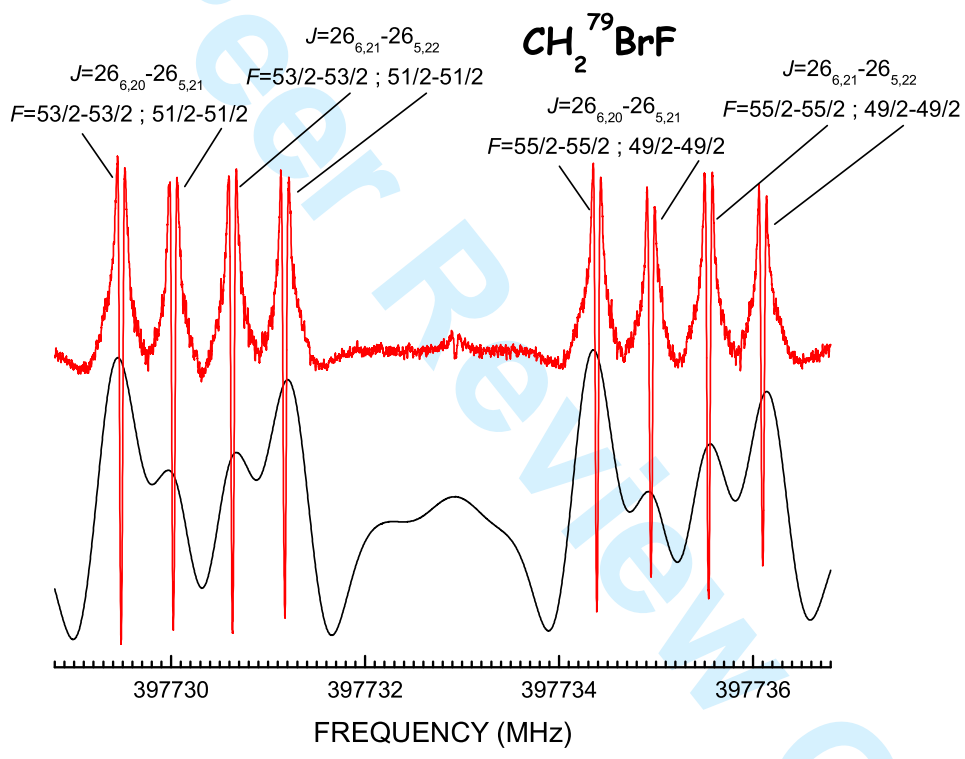

URL: http://mc.manuscriptcentral.com/tandf/tmph 


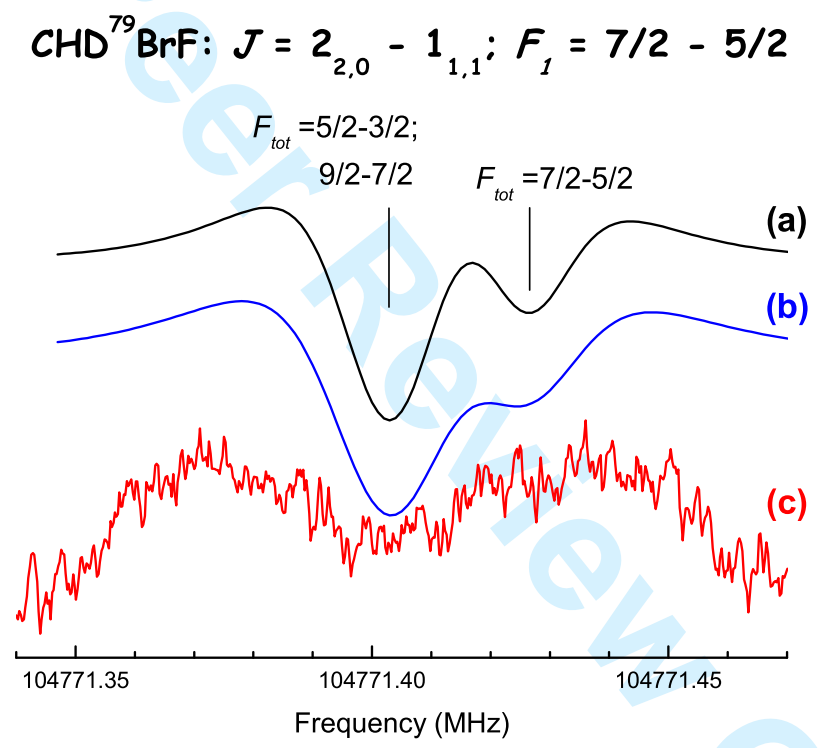

URL: http://mc.manuscriptcentral.com/tandf/tmph 